\title{
Vortex distribution in a confining potential
}

\author{
Matheus Girotto,,$^{1 *}$ Alexandre P. dos Santos, ${ }^{2, \dagger}$ and Yan Levin ${ }^{1, \ddagger}$ \\ ${ }^{1}$ Instituto de Física, Universidade Federal do Rio Grande do Sul, Caixa Postal 15051, CEP 91501-970, Porto Alegre, RS, Brazil \\ ${ }^{2}$ Departamento de Física, Universidade Federal de Santa Catarina, 88040-900 Florianópolis, Santa Catarina, Brazil
}

(Received 3 May 2013; revised manuscript received 1 July 2013; published 11 September 2013)

\begin{abstract}
We study a model of interacting vortices in a type II superconductor. In the weak coupling limit, we constructed a mean-field theory which allows us to accurately calculate the vortex density distribution inside a confining potential. In the strong coupling limit, the correlations between the particles become important and the mean-field theory fails. Contrary to recent suggestions, this does not imply failure of the Boltzmann-Gibbs statistical mechanics, as we clearly demonstrate by comparing the results of molecular dynamics and Monte Carlo simulations.
\end{abstract}

DOI: 10.1103/PhysRevE.88.032118

PACS number(s): 05.40.Fb, 05.10.Gg, 05.20.-y, 05.45.-a

\section{INTRODUCTION}

Superconductivity is one of the greatest discoveries of the previous century. The practical applications of this phenomenon rely on understanding the behavior of high temperature superconductors [1-4] in a magnetic field. Depending on the superconducting material and on external conditions, a phase with quantum magnetic vortices can appear. Superconductors with this property are called type II and are a subject of intense theoretical investigation [5-15]. The Ginzburg-Landau theory [16] predicts that the vortex-vortex interaction in a superconducting film has the form

$$
V(r)=q G\left(\mathbf{x}_{1}, \mathbf{x}_{2}\right),
$$

where

$$
G\left(\mathbf{x}_{1}, \mathbf{x}_{2}\right)=q K_{0}\left(\frac{\left|\mathbf{x}_{1}-\mathbf{x}_{2}\right|}{\lambda}\right),
$$

$K_{0}$ is a modified Bessel function, $r=\left|\mathbf{x}_{1}-\mathbf{x}_{2}\right|$ is the distance between vortex 1 and vortex $2, q$ is the vortex strength, and $\lambda$ is the London penetration length.

A number of recent papers have studied the equilibrium distribution of vortices confined by an external potential [17-19]. The authors of these papers have argued that the ground state of these systems corresponds to the maximum of the nonextensive Tsallis entropy. Contrary to this suggestion, in this paper we will present a simple mean-field theory which, in the framework of the usual Boltzmann-Gibbs (BG) statistical mechanics, accounts very well for the equilibrium distribution of vortices. Furthermore, comparing the results of molecular dynamics (MD) and Monte Carlo (MC) simulations, we will show that the system of confined vortices is described by the standard BG statistical mechanics for all the coupling strengths.

\footnotetext{
*girotto.matheus@gmail.com

†alexandre.pereira@ufrgs.br

${ }^{\ddagger}$ levin@if.ufrgs.br
}

\section{MEAN-FIELD THEORY}

We will study a system of interacting vortices confined by an external potential

$$
W(x)=\alpha x^{2} / 2 .
$$

We first observe that the function, Eq. (2), satisfies a modified Helmholtz equation

$$
\nabla^{2} G\left(\mathbf{x}, \mathbf{x}_{1}\right)-G\left(\mathbf{x}, \mathbf{x}_{1}\right)=-2 \pi q \delta\left(\mathbf{x}-\mathbf{x}_{1}\right),
$$

where all the lengths are now measured in units of $\lambda$. Consider an infinite bidimensional system of vortices in the $x-y$ plane, with periodic boundary conditions in the $y$ direction. The solution of Eq. (4) can be expressed as [20]

$$
G\left(\mathbf{x}, \mathbf{x}_{1}\right)=\frac{\pi q}{L_{y}} \sum_{m=-\infty}^{\infty} e^{\left(2 \pi m i / L_{y}\right)\left(y-y_{1}\right)} \frac{e^{-\gamma_{m}\left|x-x_{1}\right|}}{\gamma_{m}}
$$

where

$$
\gamma_{m}=\sqrt{1+4 \pi^{2} m^{2} / L_{y}^{2}}
$$

$m$ are integers and $L_{y}$ is the width of the periodic stripe in the $y$ direction.

In equilibrium, the particle distribution is given by

$$
\rho(x)=A e^{-\beta \omega(x)},
$$

where $\beta=1 / k_{B} T, \omega(x)$ is the potential of mean force (PMF), and $A$ is the normalization constant [21].

In the weak-coupling limit (high temperatures), the correlations between the vortices can be neglected and the PMF can be approximated by $\omega(x)=q \phi(x)+W(x)$. The particle distribution then becomes

$$
\rho(x)=A e^{-\beta[q \phi(x)+W(x)]},
$$

where $A$ is

$$
A=\frac{N}{L_{y} \int_{-\infty}^{+\infty} d x e^{-\beta[q \phi(x)+W(x)]}} .
$$

The potential $\phi(x)$ can be calculated using the Green's function

$$
\phi(x)=\int d \mathbf{x}^{\prime} \rho\left(x^{\prime}\right) G\left(\mathbf{x}, \mathbf{x}^{\prime}\right) .
$$




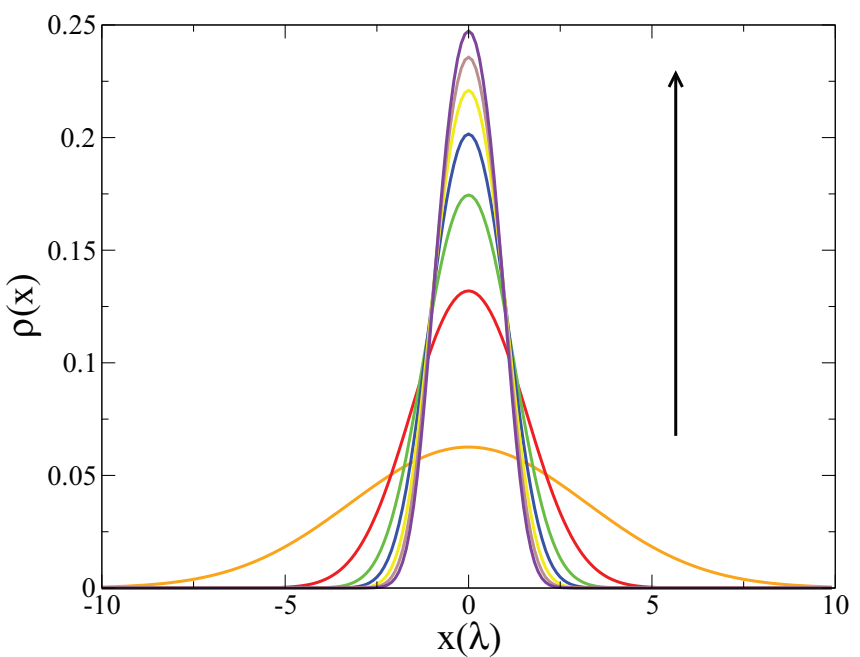

FIG. 1. (Color online) Theoretical density profiles for confined vortices. The arrow indicates increasing coupling strength (lowering temperature): $\epsilon=\chi=0.1,0.5,1,1.5,2,2.5$, and 3 .

Equation (5) allows us to rewrite Eq. (10) as

$$
\phi(x)=\pi q \int_{-\infty}^{+\infty} d x^{\prime} \rho\left(x^{\prime}\right) e^{-\left|x^{\prime}-x\right|} .
$$

The two equations (8) and (11) can be solved iteratively. To quantify the strength of the vortex-vortex and the trapvortex interaction, it is convenient to define the following
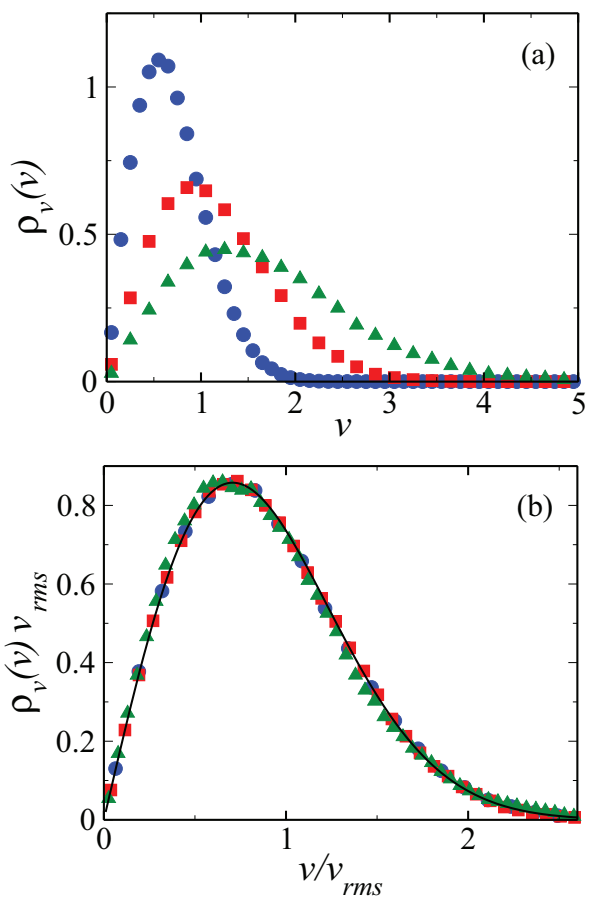

FIG. 2. (Color online) (a) Velocity distributions for various initial conditions: circles, $\epsilon=3.26$ and $\chi=1.63$; squares, $\epsilon=1.2$ and $\chi=0.6$; triangles, $\epsilon=0.54$ and $\chi=0.27$. Panel (b) shows that when the velocity is scaled with $v_{\text {rms }}$ and the distribution function is scaled with $1 / v_{\text {rms }}$, all the curves collapse onto the universal two-dimensional (2D) Maxwell-Boltzmann distribution $f(x)=2 x e^{-x^{2}}$, represented by the solid line. dimensionless parameters:

$$
\epsilon=\frac{q^{2}}{k_{B} T} \quad \text { and } \quad \chi=\frac{\alpha \lambda^{2}}{k_{B} T} .
$$

In Fig. 1 we present solutions of Eqs. (8) and (11) for various coupling parameters.
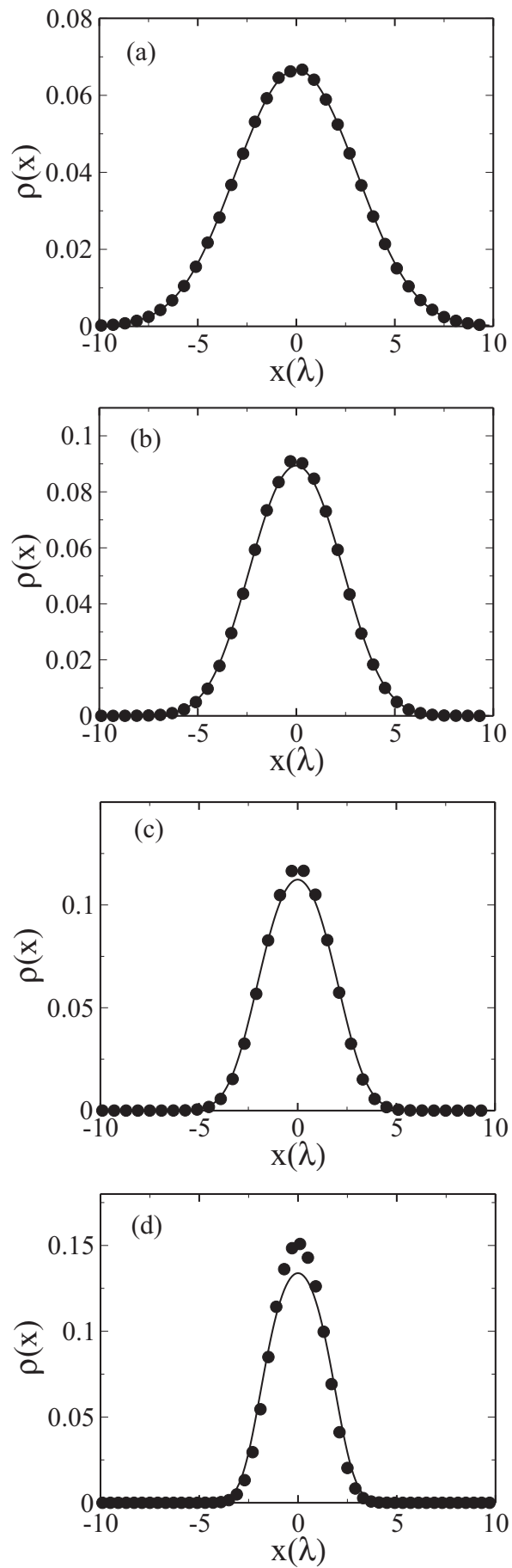

FIG. 3. Vortex density profiles: symbols represent the MD simulation data and lines are the predictions of the mean-field theory. For lower temperatures, the parameters $\epsilon$ and $\chi$ increase. The panels correspond to parameters (a) $\epsilon=0.49$ and $\chi=0.1225$, (b) $\epsilon=1$ and $\chi=0.25$, (c) $\epsilon=1.96$ and $\chi=0.49$, and (d) $\epsilon=4$ and $\chi=1$. 


\section{MOLECULAR DYNAMICS SIMULATIONS}

To verify the predictions of the mean-field theory, we first perform MD simulations. A system of $N=200$ vortices interacting by the pair potential, Eq. (1), is confined inside an infinite stripe of width $L_{y}=400$, with a trap potential $W(x)$ acting along the $x$ direction. As in Refs. [17,18], periodic boundary conditions are used in the $y$ direction. The equations of motion for each particle $i$,

$$
d^{2} \mathbf{x}_{i} / d t^{2}=-\sum_{j=1}^{N} \nabla_{\mathbf{x}_{i}} q G\left(\mathbf{x}_{i}, \mathbf{x}_{j}\right)-\alpha x_{i} \hat{\mathbf{e}}_{x},
$$

are integrated using the leapfrog algorithm.

In the simulations, a system is prepared in various initial conditions and is allowed to relax until a stationary particle distribution is established. After the equilibrium is achieved, we calculate the distribution of particle velocities, shown in Fig. 2(a). If the stationary state is the usual BG equilibrium, we expect the particle distribution to have the Maxwell-Boltzmann form, which in two dimensions is

$$
\rho_{v}(v)=\frac{2 v}{v_{\mathrm{rms}}^{2}} e^{-v^{2} / v_{\mathrm{ms}}^{2}},
$$

with $v_{\mathrm{rms}}=\sqrt{\left\langle v^{2}\right\rangle}$. This means that if the velocities are scaled with $v_{\mathrm{rms}}$ and the distribution is scaled with $1 / v_{\mathrm{rms}}$, all the curves plotted in Fig. 2(a) should collapse onto one universal curve $f(x)=2 x e^{-x^{2}}$. This is precisely what is shown to happen in Fig. 2(b). To obtain the density distribution using MD simulation, we divide the simulation stripe into bins of width $\Delta_{x}$, and calculate the average number of particles in each bin.

To compare the predictions of the mean-field theory with the results of MD simulations, we let the system relax to equilibrium and calculate the $\left\langle v^{2}\right\rangle$. For systems with short range interactions the canonical and the microcanonical ensembles must be equivalent, so that in two dimensions, $m v_{\mathrm{rms}}^{2} / 2=k_{B} T$. Using this temperature, the mean-field vortex

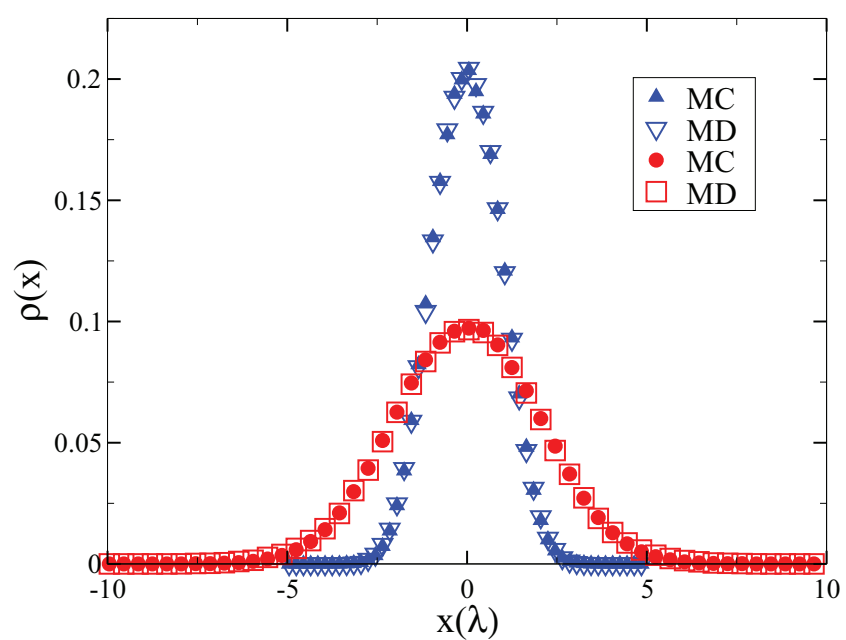

FIG. 4. (Color online) Density profiles for confined vortices obtained using MD and MC simulations: up and down triangles, $\epsilon=0.54$ and $\chi=0.27$; circles and squares, $\epsilon=3.26$ and $\chi=1.63$. $\mathrm{MC}$ and MD predict identical vortex density distributions.
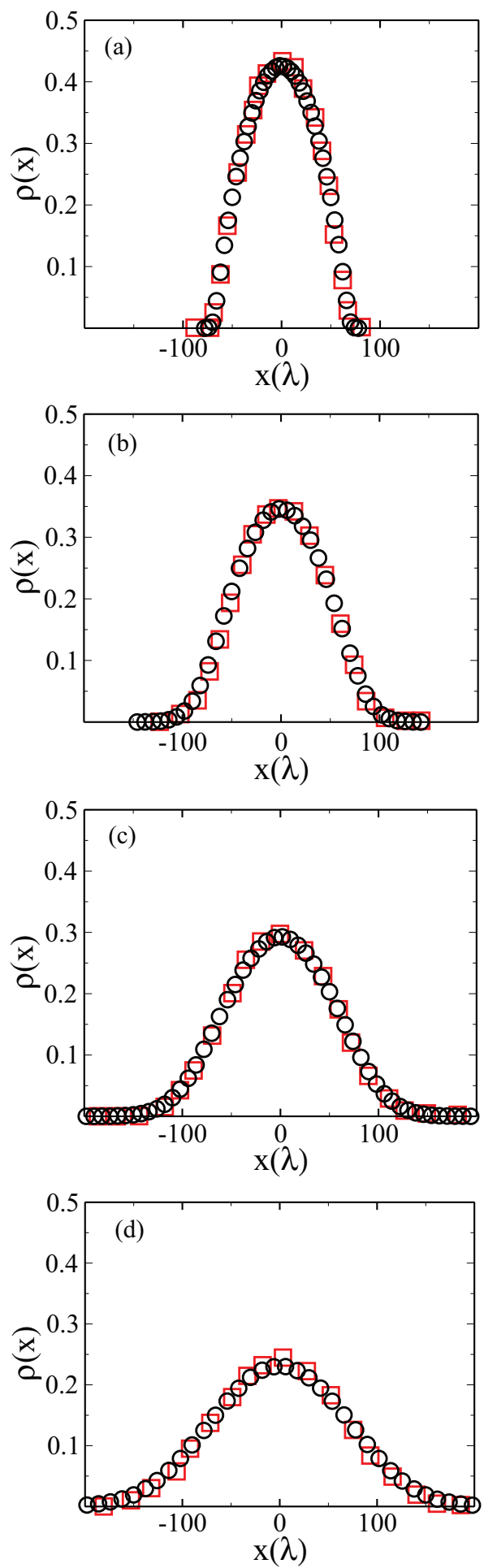

FIG. 5. (Color online) Vortex density profiles. Squares are the data of Andrade et al. from Fig. 2 of Ref. [17] and circles are the results of our MC simulations. The parameters are $\alpha=10^{-3} q^{2} / \lambda^{2}$, $N=800, L_{y}=20 \lambda$ : (a) $T=0.1 q^{2} / k_{B}$, (b) $T=1.0 q^{2} / k_{B}$, (c) $T=2.0 q^{2} / k_{B}$, and (d) $T=4.0 q^{2} / k_{B}$. The agreement between the two simulations clearly shows that the stationary state to which the system relaxes is the usual BG equilibrium.

distribution can be calculated using Eq. (8). Comparing the predictions of the mean-field theory with the results of MD 
simulations, we see that for high temperatures there is an excellent agreement; see Fig. 3. In this limit the mean-field theory, Eq. (8), becomes exact [21]. On the other hand, in the strong coupling limit (low temperatures), the correlations between the particles are important and significant deviations from the results of simulations can be seen. Correlations lead to a larger concentration of particles in the low energy states than is predicted by the mean-field theory [21]. This is similar to the process of overcharging observed in colloidal suspensions with multivalent ions [22-24].

Andrade et al. [17,18] and Ribeiro et al. [19] have argued that at low temperatures, the vortices in a type II superconductor obey Tsallis statistics (TS). In particular, they claimed that the ground state of interacting vortices in a confining potential corresponds to the maximum of the Tsallis entropy. The arguments of Andrade et al. are based on a solution of an approximate Fokker-Planck equation. This equation is very interesting and allows us to make some important predictions, such as front propagation in type II superconductors [13]. However, the fact that the stationary solution of this approximate equation at $T=0$ is a " $q$ Gaussian" does not provide justification for the relevance of the nonextensive statistical mechanics to thermodynamics of superconducting vortices. In fact, the Fokker-Planck equation for vortex density is an approximation of a more accurate Nernst-Planck-like equation, which does have the usual Boltzmann distribution as a stationary state. Neither of these equations, however, takes into account the correlations between the particles, so that both can only be valid in the mean-field limit. Nevertheless, even in this limit, Ref. [20] shows that the $q$-Gaussian solution of the Fokker-Planck equation obtained by Andrade et al. is inconsistent with the solution of the more accurate Nernst-Planck equation.

To see that the equilibrium state of the system studied by Andrade et al. is indeed described by the usual BG statistical mechanics for any temperature, we perform a series of MD and MC simulations. In MC simulations, we use the usual Metropolis algorithm [25] which is constructed to evolve the system through a Markov process towards a stationary state in which the particles are distributed (in the phase space) according to the Boltzmann distribution. Clearly if the agreement between MD and MC simulations is found, it will unequivocally show that the system of vortices interacting by the potential of Eq. (1), is both ergodic and mixing and is described by the usual BG statistical mechanics.

\section{MONTE CARLO SIMULATIONS}

We have seen already that the vortex velocity distribution is in perfect agreement with the BG statistical mechanics. In this section, we will show that the vortex density distribution is also described by the BG statistical mechanics. To do this we perform MC simulations and compare them with the results of MD simulations. MC simulations are designed to force the particles into an equilibrium state corresponding to the maximum of the Boltzmann entropy (in the microcanonical ensemble) or the minimum of the Helmholtz free energy, in the canonical ensemble. To simulate canonical ensemble one can use the Metropolis algorithm. In the Markov chain of the Metropolis algorithm, a new configuration $n$ is constructed from an old configuration $o$ by a small displacement of a random particle. The new state is accepted with a probability $P=\min \left\{1, e^{-\beta\left(E_{n}-E_{o}\right)}\right\}$, where $\beta=1 / k_{B} T$. If the movement is not accepted, the configuration $o$ is preserved and counted as a new state. The length of the displacement is adjusted during the simulation in order to obtain the acceptance rate of $50 \%$. The energy of the system used in the MC simulations is given by

$$
E=\sum_{i=1}^{N-1} \sum_{j=i+1}^{N} q G\left(\mathbf{x}_{i}, \mathbf{x}_{j}\right)+\sum_{i=1}^{N} W\left(x_{i}\right) .
$$

The Metropolis algorithm ensures that the system evolves to the $\mathrm{BG}$ thermodynamic equilibrium. The averages are calculated using $10^{5}$ uncorrelated states, obtained after $10^{6}$ MC steps for equilibration. Figure 4 shows a perfect agreement between the results of our microcanonical MD and canonical MC simulations. In Fig. 5 we compare the results of our MC simulations with the simulations of Andrade et al. (Fig. 2 of Ref. [17]) performed using an overdamped dynamics with a thermostat. Once again, the two are indistinguishable. This unequivocally demonstrates that the system of vortices, interacting by the potential of Eq. (1), is described by the usual BG statistical mechanics.

\section{CONCLUSIONS}

We have studied a simple model of interacting vortices in a type II superconductor. In the weak coupling limit we have constructed a mean-field theory which allows us to accurately calculate the equilibrium vortex density distribution inside a confining potential. In the strong coupling limit the correlations between the particles become important and the mean-field theory fails. This, however, does not imply the failure of the BG statistics, as is clearly demonstrated by the perfect agreement between MD and MC simulations and by the Maxwell-Boltzmann distribution of the particle velocities.

It is very difficult to study theoretically the correlations in inhomogeneous liquids. A number of different approaches, such as density functional theory (DFT) [26-30] and integral equations [31,32], have been developed over the years. All these theoretical methods are firmly embedded in the framework of the BG statistics. Introduction of novel type of entropies [17-19] as a way to fit in the interparticle correlations does not help to shed new light on the equilibrium properties of these interesting systems.
[1] A. F. Hebard, M. J. Rosseinsky, R. C. Haddon, D. W. Murphy, S. H. Glarum, T. T. M. Palstra, A. P. Ramirez, and A. R. Kortan, Nature (London) 350, 600 (1991).

[2] G. Blatter, M. V. Feigelman, V. B. Geshkenbein, A. I. Larkin, and V. M. Vinokur, Rev. Mod. Phys. 66, 1125 (1994).
[3] E. H. Brandt, Rep. Prog. Phys. 58, 1465 (1995).

[4] J. Nagamatsu, N. Nakagawa, T. Muranaka, Y. Zenitani, and J. Akimitsu, Nature (London) 410, 63 (2001).

[5] H. J. Jensen, A. Brass, and A. J. Berlinsky, Phys. Rev. Lett. 60, 1676 (1988) 
[6] O. Pla and F. Nori, Phys. Rev. Lett. 67, 919 (1991).

[7] M. W. Coffey and J. R. Clem, Phys. Rev. Lett. 67, 386 (1991).

[8] V. V. Bryksin and S. N. Dorogovtsev, Physica C 215, 173 (1993).

[9] V. V. Bryksin and S. N. Dorogovtsev, Zh. Eksp. Teor. Fiz. 104, 3735 (1993).

[10] R. A. Richardson, O. Pla, and F. Nori, Phys. Rev. Lett. 72, 1268 (1994).

[11] M. W. Coffey, Phys. Rev. B 54, 1279 (1996).

[12] W. Barford, Phys. Rev. B 56, 425 (1997).

[13] S. Zapperi, A. A. Moreira, and J. S. Andrade, Jr., Phys. Rev. Lett. 86, 3622 (2001).

[14] M. E. Zhitomirsky and V. H. Dao, Phys. Rev. B 69, 054508 (2004).

[15] P. J. Lin and P. Lipavsky, Phys. Rev. B 80, 212506 (2009).

[16] P. G. de Gennes, Superconductivity of Metals and Alloys (Addison-Wesley, Redwood City, CA, 1989).

[17] J. S. Andrade, Jr., G. F. T. da Silva, A. A. Moreira, F. D. Nobre, and E. M. F. Curado, Phys. Rev. Lett. 105, 260601 (2010).

[18] J. S. Andrade, Jr., G. F. T. da Silva, A. A. Moreira, F. D. Nobre, and E. M. F. Curado, Phys. Rev. Lett. 107, 088902 (2011).
[19] M. S. Ribeiro, F. D. Nobre, and E. M. F. Curado, Phys. Rev. E 85, 021146 (2012).

[20] Y. Levin and R. Pakter, Phys. Rev. Lett. 107, 088901 (2011).

[21] Y. Levin, Rep. Prog. Phys. 65, 1577 (2002).

[22] A. Y. Grosberg, T. T. Nguyen, and B. I. Shklovskii, Rev. Mod. Phys. 74, 329 (2002).

[23] S. Pianegonda, M. C. Barbosa, and Y. Levin, Europhys. Lett. 71, 831 (2005).

[24] A. P. dos Santos, A. Diehl, and Y. Levin, J. Chem. Phys. 132, 104105 (2010).

[25] N. Metropolis, A. W. Rosenbluth, M. N. Rosenbluth, A. H. Teller, and E. Teller, J. Chem. Phys. 21, 1087 (1953).

[26] P. Tarazona, Phys. Rev. A 31, 2672 (1985).

[27] W. A. Curtin and N. W. Ashcroft, Phys. Rev. A 32, 2909 (1985).

[28] A. R. Denton and N. W. Ashcroft, Phys. Rev. A 39, 4701 (1989).

[29] R. D. Groot, J. Chem. Phys. 95, 9191 (1991).

[30] A. Diehl, M. N. Tamashiro, M. C. Barbosa, and Y. Levin, Physica A 274, 433 (1999).

[31] R. Kjellander, J. Chem. Phys. 88, 7129 (1988).

[32] R. Kjellander, J. Chem. Phys. 89, 7649(E) (1988). 